\title{
LIMITING CURRENT SINGULARITIES IN ELECTRO DIFFUSION
}

\author{
BY \\ C. DUBI
}

Department of Mathematics, Ben-Gurion University of the Negev, Beer-Sheva, 84105, Israel

\begin{abstract}
This paper concerns the phenomenon of limiting current in the local electro neutrality approximation of electrodiffusion. The limiting current is the value of the control parameter, the electric current, for which the straightforward split of an electrodiffusional system into a quasi-electro-neutral bulk and charged boundary (electric double) layer valid near the equilibrium is no longer expected to stand. Singularities in the solution appearing at the limiting current are studied.
\end{abstract}

1. Introduction. The system of differential equations, describing the one-dimensional steady state electrodiffusion in an electrolyte solution, in dimensionless form reads:

$$
\begin{gathered}
\epsilon \phi^{\prime \prime}=N-p+n \\
\left(n^{\prime}-\phi^{\prime} n\right)^{\prime}=0 \\
\left(p^{\prime}+\phi^{\prime} p\right)^{\prime}=0 .
\end{gathered}
$$

Upon integration of the last two equations we have

$$
\begin{aligned}
& n^{\prime}-\phi^{\prime} n=-j_{-} \\
& p^{\prime}+\phi^{\prime} p=-j_{+}
\end{aligned}
$$

Here, the unknowns $n(x), p(x)$ are the anionic and cationic concentrations respectively, $\phi(x)$ is the electric potential, and $N$ is a given function of $x$, specifying the concentration of fixed charges in the system. The usually unknown integration constants $j_{-}, j_{+}$are the ionic fluxes. Their difference $I \stackrel{\text { def }}{=} j_{+}-j_{-}$is the electric current. When specified by the boundary conditions, $I$ constitutes a natural control parameter.

In macroscopic electrodiffusional systems the constant $\epsilon$ is in the range $10^{-12} \leq \epsilon \leq$ $10^{-8}$. Thus, the problem under consideration is a singular perturbation problem. The standard approach to such problems is via the boundary layer method. In the latter, the interval of solution is divided into the "bulk" (domain where the solution is governed by the unperturbed equation) and the boundary layer, where the solution is governed by a different equation, obtained by stretching the independent variable.

Received September 10, 2001.

2000 Mathematics Subject Classification. Primary 34Exx. 
A prototypical unperturbed electrodiffusional boundary value problem reads:

$$
\begin{gathered}
N=p-n \\
n^{\prime}-\phi^{\prime} n=-j_{-} \\
p^{\prime}+\phi^{\prime} p=-j_{+}
\end{gathered}
$$

with the boundary conditions

$$
\begin{gathered}
j_{+}-j_{-}=I \\
n(-1)=n^{-}>0 \quad n(1)=n^{+}>0 \\
p(-1)=p^{-}=n^{-}+N(-1)>0 \quad p(1)=p^{+}=n^{+}+N(1)>0 .
\end{gathered}
$$

Here $I, n^{ \pm}, p^{ \pm}$are given constants. Boundary conditions $(1.3 \mathrm{~b}, 1.3 \mathrm{c})$ are such as to avoid boundary layers at $x= \pm 1$.

We are interested in situations where the unperturbed system fails to approximate the perturbed problem. An obvious instance of this would be the concentrations $n(x)$ or $p(x)$ becoming negative, for the values of the control parameter-electric current above a certain threshold. This threshold value is termed the limiting current (see $[1,2,4])$.

It is the main purpose of this paper to show that reaching the limiting current is accompanied by what may be looked upon as a "breakdown" of the solution, namely, the appearance of singularities in the solution for an analytic fixed charge concentration $N(x)$.

This paper has the following structure. In the next section we make the necessary definitions and prove a few simple facts concerning the limiting current. In the third section we study singularities in the solution appearing at the limiting current.

\section{Limiting Current.}

Definition 2.1. In boundary value problem (1.2) (1.3), a current $I$ is said to be non-physical if for every $\tilde{I}$ such that:

1. $|\tilde{I}>| I \mid$

2. $\operatorname{sign}(I)=\operatorname{sign}(\tilde{I})$

there exists $x \in[-1,1]$ such that $n(x)=0$ or $p(x)=0$.

Let us denote

$$
\Omega^{+}=\{I>0 \mid I \text { is non-physical }\} \quad \Omega^{-}=\{I<0 \mid I \text { is non-physical }\} .
$$

Definition 2.2. A current $I$ is said to be a limiting current if $I=\inf \Omega^{+}$or $I=$ $\sup \Omega^{-}$.

Notice that according to definition (2.2), the limiting current does not have to be unique. Rather, there may be two limiting currents at most: a positive and a negative one.

As pointed out in previous studies $[2,3]$, the limiting current may be viewed as a current value above which the straightforward use of boundary layer method fails in the perturbed problem, and, thus, has to be modified. Such a modification is provided in terms of the respective free boundary formulation. 
Proposition 2.3. Let $(n(x), p(x), \phi(x))$ be a solution to (1.2) with boundary conditions (1.3), and $N(x) \in C^{1}$. Assume that $n(x), p(x) \geq 0$. If there exists $\tilde{x} \in[-1,1]$ such that $n(\tilde{x})=0$ or $p(\tilde{x})=0$, then

$$
n(\tilde{x})=p(\tilde{x})=N(\tilde{x})=0 .
$$

Proof. First we define the auxiliary function

$$
\sigma(x)=n(x)+p(x)
$$

Using (2.1), equations $(1.2 \mathrm{a}, 1.2 \mathrm{~b})$ are rewritten as:

$$
\begin{gathered}
\sigma^{\prime}+N \phi^{\prime}=-I-2 j_{-} \\
N^{\prime}+\sigma \phi^{\prime}=-I .
\end{gathered}
$$

Thus, for $\sigma(x)$ we have:

with boundary conditions

$$
\sigma^{\prime}=\frac{N\left(N^{\prime}+I\right)}{\sigma}+J
$$

$$
\begin{gathered}
\sigma(-1)=\sigma^{-} \stackrel{\text { def }}{=} n^{-}+p^{-}>0 \\
\sigma(1)=\sigma^{+} \stackrel{\text { def }}{=} n^{+}+p^{+}>0 .
\end{gathered}
$$

Here $J=j_{+}+j_{-}=I+2 j_{-}$. Notice that while $I$ is specified by the boundary conditions, $J$ is an unknown to be determined as a part of the solution.

Let us assume, without loss of generality, that $n(\tilde{x})=0$. From (1.2) and (2.1) we obtain $\sigma(\tilde{x})=p(\tilde{x})=N(\tilde{x})$. Furthermore, let us assume, to arrive at a contradiction, that $\sigma(\tilde{x})=p(\tilde{x})=N(\tilde{x}) \neq 0$. Equation (2.3) then yields

$$
\sigma^{\prime}(\tilde{x})=N^{\prime}(\tilde{x})+I-J
$$

Since $n(x), p(x) \geq 0$, we conclude, using (1.2), that $\sigma(x) \geq N(x)$. Since $\sigma(\tilde{x})=p(\tilde{x})=$ $N(\tilde{x}), N(x)$ and $\sigma(x)$ are tangent at $\tilde{x}$. Thus, by (2.3) we have $J=I$. The latter equality implies $j_{-}=0$. Equation (1.2) then yields, upon integration:

$$
n(x)=C e^{-\phi}, \quad C=\text { const } .
$$

Since, by the boundary conditions $n(x)$ is not identically $0, C \neq 0$. Therefore, by $(2.6)$ we have $\lim _{x \rightarrow \tilde{x}}|\phi(x)|=\infty$. Due to (2.2), this implies $\sigma(\tilde{x})=0$. Thus, $n(\tilde{x})=p(\tilde{x})=0$, in contradiction to our assumption.

Definition 2.4. Boundary value problem (2.3), (2.4) is said to be neutral if

$$
\int_{-1}^{1} N(x) d x=0
$$

DEFinition 2.5. Boundary value problem (2.3), (2.4) is said to be symmetric if

$$
\left(\sigma^{+}\right)^{2}-\left(\sigma^{-}\right)^{2}-\left(N(1)^{2}-N(-1)^{2}\right)-2 I \int_{-1}^{1} N(x) d x=0
$$

Proposition 2.6. For a given symmetric problem $(2.3),(2.4)$ with a solution $\sigma(x) \not \equiv 0$, $J$ vanishes. 
Proof. Multiplying both sides of Eq. (2.3) by $\sigma(x)$, followed by integration over the interval $[-1,1]$, yiclds:

$$
\left(\sigma^{+}\right)^{2}-\left(\sigma^{-}\right)^{2}-(N(1))^{2}-(N(-1))^{2}=2 I \int_{-1}^{1} N(x) d x-2 J \int_{-1}^{1} \sigma(x) d x .
$$

Since $\sigma$ is continuous and not identically zero, $\int_{-1}^{1} \sigma(x) d x \neq 0$. Using the symmetry condition (2.8) we conclude that $2 J \int_{-1}^{1} \sigma(x) d x=0$, that is, $J=0$.

Let us notice the following two facts:

1. In a neutral system, the symmetric condition does not contain $I$.

2. Once $J=0$, the initial value problem (2.3). (2.4b) may be explicitly solved by separation of variables, yiclding

$$
\sigma(x)=\sqrt{N^{2}(x)+2 I \int_{-1}^{x} N(t) d t+\left(\left(\sigma^{+}\right)^{2}-(N(-1))^{2}\right)} .
$$

If the problem is neutral as well, solution (2.10) is valid for an arbitrary $I$ which is not the case in the general non-neutral case.

One may easily see that in this case $\sigma(x)$ is continuous and point-wise monotonic in $I$, increasing if $\int_{-1}^{x} N(t) d t>0$ and decreasing if $\int_{-1}^{x} N(t) d t<0$. Thus, the criterion for the limiting current $I^{\mathrm{lim}}$ is the existence of a point $\tilde{x} \in[-1,1]$ such that: $\sigma\left(\tilde{x} \mid I^{\mathrm{lim}}\right)=|N(\tilde{x})|$. Using Proposition 2.3 we conclude that $\sigma\left(\tilde{x} \mid I^{\lim }\right)=|N(\tilde{x})|=0$. Since $N(x)$ is analytic, it has a finite number of roots, which yields the following formula for the limiting current:

Proposition 2.7. Let $\sigma(x \mid I)$ be the solution (2.10) to symmetric neutral boundary value problem $(2.3),(2.4)$. Let $a_{1}, a_{2}, \ldots, a_{n}$ be the roots of $N(x)$. Then the limiting current $I^{\lim }$ is:

$$
I^{\lim }=\min \left\{I>0 \mid \exists i \in 1,2, \ldots n ; 2 I \int_{-1}^{a_{i}} N(t) d t=-\left(\left(\sigma^{+}\right)^{2}-(N(-1))^{2}\right)\right\}
$$

or:

$$
I^{\lim }=\max \left\{I<0 \mid \exists i \in 1,2, \ldots n ; 2 I \int_{-1}^{a_{i}} N(t) d t=-\left(\left(\sigma^{+}\right)^{2}-(N(-1))^{2}\right)\right\} .
$$

Proof. The proof follows from Eq. (2.10) and Definition 2.2.

Equations (2.11), (2.12) may be found in [4] but for a different definition of the limiting current, and $N(x)$ piecewise constant, whereas here it is analytic.

3. Singularities Related to the Limiting Current. In the last section a formula was given for the limiting current in a neutral symmetric system. Let us now assume that all the roots of $N(x)$ are simple. In this case $|N(x)|$ is not differentiable at its roots. Since for $I=I^{\lim }$ we have that $|N(x)|<\sigma(x)$ for every $x \in[-1,1]$ which is not a root of $N(x)$ and $N(\tilde{x})=\sigma(\tilde{x})=0$ for a root $\tilde{x}$ of $N(x)$, clearly, $\sigma$ is not differentiable at $\tilde{x}$. For the sake of simplicity, $\tilde{x}$ will denote from here on the point in the interval $[-1,1]$ where $\sigma\left(x \mid I^{\text {lim }}\right)$ vanishes.

From (2.10), for every physical current, $\sigma(x \mid I)$ is a non-vanishing analytic function, and thus an analytic function itself. Since $\sigma(x \mid I)$ is pointwise monotonic, we conclude that for the aforementioned $N(x)$ the limiting current is characterized by the appearance 
of discontinuities in the first derivative of $\sigma(x \mid I)$ at $\tilde{x}$. The latter is accompanied by an unbounded growth of the electric field at $\tilde{x}$, which is the feature traditionally associated with the limiting current (see $[4,1,2])$.

Indeed, by Eq. (2.2):

$$
\phi^{\prime}=\frac{-I-N^{\prime}}{\sigma}
$$

and, generally, in the neutral symmetric case $I^{\text {lim }} \neq-N^{\prime}(\tilde{x})$, whereas $\sigma(\tilde{x})=0$ for $I=I^{\text {lim }}$. Let us allow now for multiple roots of $N(x)$, still, for a neutral symmetric case (dropping the latter assumption would require a reference to continuity of the solution with respect to the current, which has not been proved thus far). Even for a neutral symmetric system, we do not have a complete description of the limiting current singularities for a general $N(x)$ with multiple roots. On the other hand, some understanding may be inferred from the prototypical examples below.

Recall, first, that by $(2.10)$ we have:

$$
\sigma(x)=\sqrt{N^{2}(x)+2 I \int_{\tilde{x}}^{x} N(t) d t+\delta} .
$$

Here $\delta=\sigma^{2}(\tilde{x})$.

For $N^{\prime}(\tilde{x})=0$, it is still possible that $\sigma^{\prime}(\tilde{x})=0$ and no discontinuity appears in the first derivative whenever the following condition holds:

$$
\lim _{x \rightarrow \tilde{x}} \sigma^{\prime}(x)=\lim _{x \rightarrow \tilde{x}} \frac{2 N N^{\prime}+2 I^{\lim } N}{\sqrt{N^{2}(x)+2 I \int_{\tilde{x}}^{x} N(t) d t+\delta}} .
$$

Still, in this case singularity might appear in higher orders, as illustrated by the following examples, all for the interval $[-1,1]$.

EXAMPLE 3.1. $N(x)=x$.

In this case, by (3.14) we have

$$
\sigma(x)=\sqrt{x^{2}+2 I^{\lim } \int_{0}^{x} t d t}=|x| \sqrt{I^{\lim }+1} .
$$

As mentioned previously, the solution is continuous but not its first derivative.

EXAMPLE 3.2. $N(x)=x^{3}$.

In this case

$$
\sigma(x)=\sqrt{x^{6}+\frac{I^{\lim }}{2} x^{4}}
$$

and by direct differentiations:

$$
\begin{gathered}
\sigma^{\prime}(x)=\frac{3 x^{5}+I^{\lim } x^{3}}{\sqrt{x^{6}+\frac{I^{\lim }}{2} x^{4}}} \\
\sigma^{\prime \prime}(x)=\frac{\left(15 x^{5}+3 I^{\lim } x^{2}\right)\left(x^{6}+\frac{I^{\lim }}{2} x^{4}\right)-\left(3 x^{5}+I^{\lim } x^{3}\right)}{\left(x^{6}+\frac{I^{\lim }}{2} x^{4}\right)^{3 / 2}} .
\end{gathered}
$$


Considering the limit $x \rightarrow 0$, we obtain from the latter

$$
\begin{aligned}
\lim _{x \rightarrow 0^{+}} \sigma^{\prime \prime}(x)= & \lim _{x \rightarrow 0^{-}} \sigma^{\prime \prime}(x)=\frac{1.5\left(I^{\lim }\right)^{2}-\left(I^{\mathrm{lim}}\right)^{2}}{\left(0.5 I^{\lim }\right) 1.5}=\sqrt{2 I^{\lim }} \\
& \lim _{x \rightarrow 0} \sigma(x)=\sqrt{0.5^{\lim } x^{2}+o\left(x^{3}\right) .}
\end{aligned}
$$

Thus, $\sigma(x)$ is twice continuously differentiable with a discontinuous third derivative.

EXAMPLE 3.3. $N(x)=x^{5}$.

Once more, by differentiation, we observe that $\sigma(x)$ is twice continuously differentiable, whereas $\lim _{x \rightarrow 0^{+}} \sigma^{(3)}(x)=\frac{2}{\sqrt{3}} \sqrt{I^{\lim }}=-\lim _{x \rightarrow 0^{-}} \sigma^{(3)}(x) \neq 0$. Again, this was anticipated, since

$$
\lim _{x \rightarrow 0} \sigma^{(3)}(x)=\left|x^{3}\right| \sqrt{\frac{1}{3} I^{\lim }}+o\left(x^{5}\right) .
$$

These examples suggest the following conjecture:

Conjecture 3.4. For $N(x)$ analytic in the interval $[-1,1]$ with a root of multiplicity $2 k+1$ at $x=0, \sigma(x)$, a solution of a neutral symmetric boundary value problem $(2.3),(2.4)$, is:

1. $2 k$ times continuously differentiable, with a discontinuous $2 k+1$ derivative, for $k$ odd;

2. $k$ times continuously differentiable, with a discontinuous $k+1$ derivative, for $k$ even.

Although we have no rigorous proof of this conjecture, the possible heuristic idea behind it might be as follows:

According to (3.14), for $N(x)$ of the aforementioned type, we have:

$$
\sigma(x)=\sqrt{a x^{4 x+2}+o(4 x+3)+b x^{2 x+2}+o(2 x+3)} .
$$

For $k$ odd, a singularity follows from the term $a x^{4 x+2}$ (since the square root of $b x^{2 x+2}$ is analytic at 0 ), whereas for $k$ even. the singularity is determined by the term $b x^{2 x+2}$.

4. Conclusions. The described singularities, developing at the limiting current, may be viewed, in a quasi-electro-neutral electrodiffusional system with an analytic distribution of fixed charges, as precursors of the free boundary and their related discontinuities of charge carrier concentrations which are expected to develop for currents above the limiting value. Dynamics of such free boundary have been described for systems with piecewise constant fixed charge distribution $[1,2,3]$. Such study for an analytic fixed charge distribution, to the best of our knowledge, remains to be done.

Acknowledgement. The author is grateful to Isalak Rubinstein for introduction to the subject and valuable discussions.

\section{References}

[1] I. Rubinstein, Electro diffusion of ions, SIAM, 1990

[2] M. Primicerio, I. Rubinstein, and B. Zaltsman, Electrodiffusional free boundary problem in a bipolar membrane at a reverse bias for constant current, Quart. Appl. Math. 57, 637 (1999)

[3] I. Rubinstein and B. Zaltsman. Electrodiffusional free boundary problem in concentration polarization in electrodialysis, Mathematical Models and Methods in Applied Science 6, 263 (1996) 
[4] F. Alabau, Structural properties of the one-dimensional drift diffusion models for semiconductors, Trans. Amer. Math. Soc., 3486 (1996) 